\title{
First application of the 3D-MHB on dynamic compressive behavior of UHPC
}

\author{
Ezio Cadoni ${ }^{1}$, a, Matteo Dotta ${ }^{1}$, Daniele Forni ${ }^{1,2}$, Gianmario Riganti $^{1}$, and Carlo Albertini ${ }^{3}$ \\ ${ }^{1}$ DynaMat Laboratory, University of Applied Sciences of Southern Switzerland, 6952 Canobbio, Switzerland \\ ${ }^{2}$ Department of Structural, Geotechnical and Building Engineering - Politecnico di Torino, 10129 Torino, Italy \\ ${ }^{3}$ Dynalab-Impact Technologies, 21014 Laveno Mombello, Italy
}

\begin{abstract}
In order to study the dynamic behaviour of material in confined conditions a new machine was conceived and called 3D-Modified Hopkinson Bar (3D-MHB). It is a Modified Hopkinson Bar apparatus designed to apply dynamic loading in materials having a tri-axial stress state. It consists of a pulse generator system (with pre-tensioned bar and brittle joint), 1 input bar, and 5 output bars. The first results obtained on Ultra High Performance Concrete in compression with three different mono-axial compression states are presented. The results show how the pre-stress states minimize the boundary condition and a more uniform response is obtained.
\end{abstract}

\section{Introduction}

Loading rate has effects on brittle material fracturing mechanism and mechanical properties including strength and absorbed energy.

The rate dependent behaviour of the brittle material in compression may be influenced by many factors including porosity, water content, boundary condition at the interface (specimen - bar) and confining pressure.

Understanding the dynamic response of Ultra High Performance Concrete (UHPC) has important applications on engineering, including protective structures and critical infrastructures $[1,2]$.

The materials in a real structure [3,4] are usually subjected to a service load and often are also subjected to confinement. Dynamic loads (such as explosion, earthquake or impact) are loads applied to the structures together with the existing in situ stresses. Therefore, the test conditions should be as similar as possible to the actual conditions. This is the reason why a mono-, bi-, or tri-axial static stress state is applied before to the impact loading.

In order to study the dynamic behaviour of material in confined conditions a new machine was conceived and called 3D-Modified Hopkinson Bar (3D-MHB). It is a Modified Hopkinson Bar apparatus designed to apply dynamic loading in materials having a tri-axial stress state. It consists of a pulse generator (the pulse is generated by energy stored in a pre-tensioned bar and relaxed by the rupture of a fragile bolt), 1 input bar, and 5 output bars. The output bars are also providing static stresses to create a static tri-axial compression at required level.

Waves are measured by strain gauges on the input and all 5 output bars, to provide stress and strain.

The main innovation of the 3D-MHB apparatus is in the possibility to study the dynamic effects in confined materials (important for rocks, concrete, etc.). In fact, since 3 independent pairs of pressure bars will be used,

\footnotetext{
${ }^{a}$ Corresponding author: ezio.cadoni@supsi.ch
}

the lateral confinement effect to the dynamic strength of materials can be investigated. The confinement effect is very crucial to brittle material failure. The confinement effect in dynamic conditions at high strain rates has not been experimentally examined largely due to the limitation of the existing equipment. This equipment was proposed in [5] and developed in the frame of the R'Equip program of the Swiss National Sciences Foundation [6]. This is innovative equipment and is the first piece of such kind of research equipment worldwide. The equipment was designed for a comprehensive use in future researches on dynamics of materials.

The theme tackled in this paper is the presentation of the first results obtained on UHPC in dynamic compression with three different mono-axial compression states.

\section{Materials}

The UHPC studied in this first application was the matrix of a commercial UHPFRC product [7]. It was a cementitious composite obtained mixing cement, water, quartz sand, micro-silica, and super plasticizers. The UHPFRC is usually obtained by reinforcing this composite with an amount of high strength steel fibres. The tests were carried out on cylindrical specimens having a diameter of $30 \mathrm{~mm}$ and height of $30 \mathrm{~mm}$.

The quasi-static compressive strength was measured on three specimens by means of an electro-mechanical universal machine having $680 \mathrm{kN}$ maximum load capacity obtaining a compressive strength of $f_{c}=172 \pm 9 \mathrm{MPa}$. The UHPFRC with $3 \%$ of fibre content had a compressive strength of $212 \mathrm{MPa}$.

\section{The 3D-MHB apparatus}

The 3D-MHB is a Hopkinson bar apparatus able to apply a quasi-static tri-axial stress before the dynamic test. The basic concept is shown in Fig. 1. It consists of one 


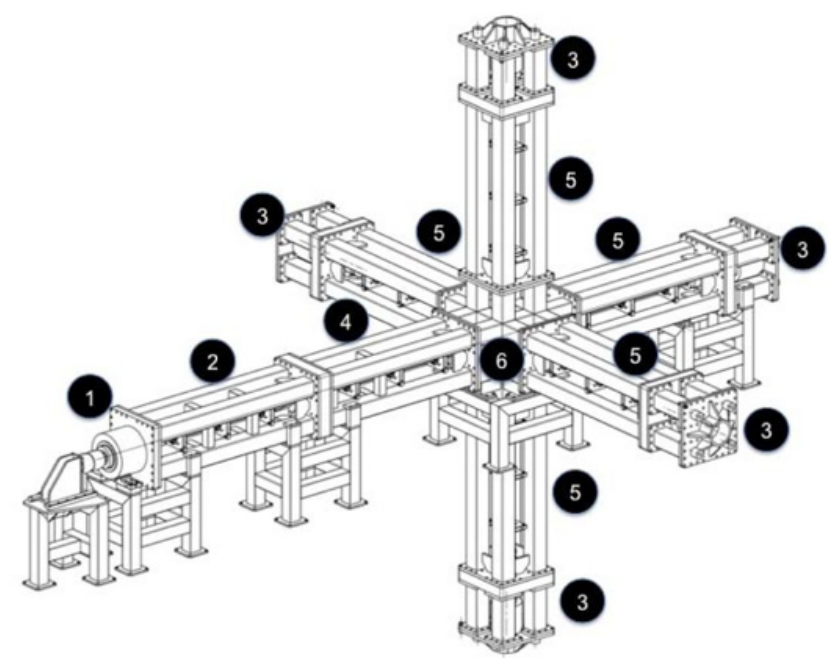

Figure 1. 3D-MHB apparatus scheme.

Table 1. Dimension of the 3D-MHB.

\begin{tabular}{|c|c|c|c|}
\hline Element & $\begin{array}{c}\text { dimension } \\
{[\mathbf{m m}]}\end{array}$ & $\begin{array}{c}\text { area } \\
{\left[\mathbf{m m}^{\mathbf{2}}\right]}\end{array}$ & $\begin{array}{c}\text { length } \\
{[\mathbf{m m}]}\end{array}$ \\
\hline $\begin{array}{c}\text { Pre-tensioned bar } \\
\text { (round) }\end{array}$ & $\varnothing=56.5$ & 2507 & 1750 \\
\hline Input bar (square bar) & $\mathrm{L}=50$ & 2500 & 2200 \\
\hline $\begin{array}{c}\text { Output bar } \\
\text { (square bar) }\end{array}$ & $\mathrm{L}=50$ & 2500 & 2100 \\
\hline
\end{tabular}

hydraulic actuator (1) connected to the pre-tensioned bar (2), and of five other hydraulic actuators (3) installed at the end of the five output bars (5). Finally the input bar (4) is connected directly to the pre-tensioned bar in one end and in the other end is in contact with the specimen (6).

A quasi-static tri-axial stress state is introduced in the specimen by the hydraulic actuators (3) of the outputconfinement bars (5). The rupture of the brittle bolt (between (1) and (2)) gives rise to a rectangular square pulse propagating into the system and loading dynamically the specimen until fracture. The strain gauges on the input (4) and output-confinement bars (5) record the incident, reflected and transmitted pulses allowing the reconstruction of the equivalent stress-strain curves.

It is important to realise that the measurements on all the output bars also provide information on wave/energy transmission and transformation at material failure. This allows to study energy/wave released during material fracturing.

\subsection{The uniaxial set-up of 3D-MHB}

The construction of the 3D-MHB apparatus started [6] with the uniaxial set-up. The new equipment was built and installed in the DynaMat Laboratory. In this configuration it represents a Modified Hopkinson Bar in compression with the addition of a hydraulic actuator at the end of the output bar which has the function of imposing the static pre-stress to the specimen. It consists of a pretensioned bar (cylindrical bar), input and output bars (with square cross-section). The dimensions of the three bars

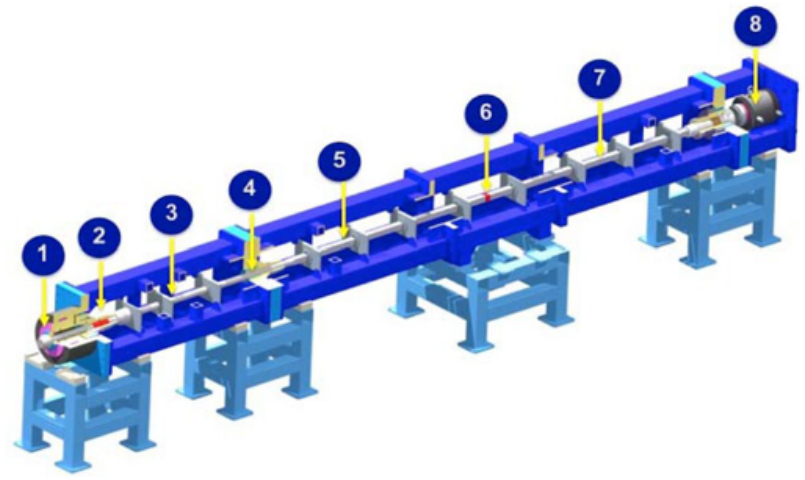

Figure 2. Experimental set-up for mono-axial testing.

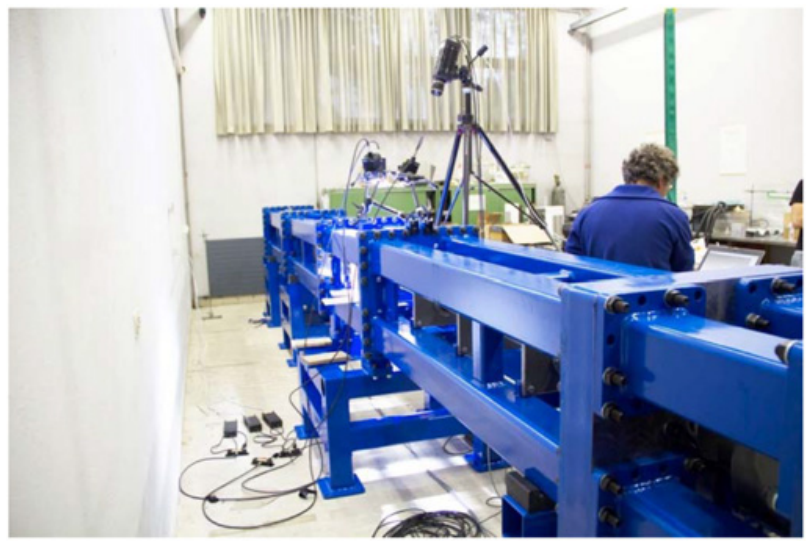

Figure 3. 3D-MHB set-up view.

are reported in Table 1. The total length of the 3D-MHB apparatus along the impact axis is of $7.82 \mathrm{~m}$. The length becomes $8.80 \mathrm{~m}$ when the bumper system is installed.

All bars are made of aged maraging steel. This material has several advantages compared to other high strength steels. First, it is possible to reach the required strength, which is $1^{\prime} 600 \mathrm{MPa}$ in the measurement cross-section and locally higher at connections and other discontinuities. Than, the ageing treatment requires moderate heating (around $500{ }^{\circ} \mathrm{C}$ ) and slow cooling minimizing thermal induced stress and thermal deformation of the pieces. It is possible to produce the bars with tiny details like threads without machining after ageing. It is also possible to treat the bars and the counter pieces to reach slightly different strength, minimizing the cost in case of damage. The drawbacks of the maraging steel are the cost and the limited choice of raw dimensions.

The 3D-MHB apparatus can generate a rectangular loading pulse of $3 \mathrm{MN}$ amplitude and $0.800 \mathrm{~ms}$ duration which propagates through the input bar - specimen output bar. It deforms the specimen at high strain rate up to fracture.

The operative sequence in order to carry out a test using the $3 \mathrm{D}-\mathrm{MHB}$ is as follows (refers to Fig. 2):

(i) A quasi-static stress state is introduced in the UHPC specimen by the hydraulic actuator placed at the end of the output bar (8). 
Table 2. Dynamic compressive strength of UHPC.

\begin{tabular}{|c|c|}
\hline Preload [kN] & $\begin{array}{c}\text { Dynamic compressive } \\
\text { strength [MPa] }\end{array}$ \\
\hline 0 & $456 \pm 65$ \\
\hline 40 & $504 \pm 25$ \\
\hline 80 & $455 \pm 12$ \\
\hline
\end{tabular}

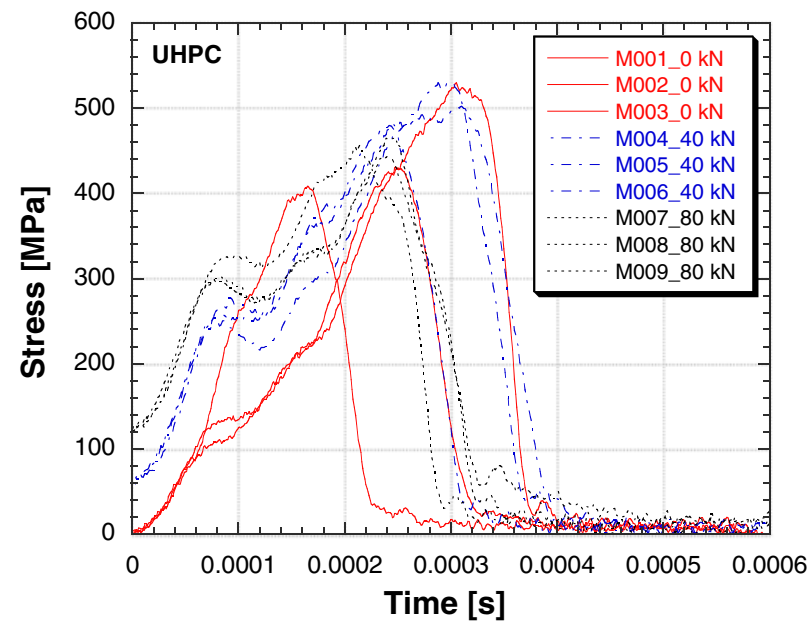

Figure 4. Stress versus time curves of UHPC with different pre-loading states.

(ii) By the principal hydraulic actuator (1) the pretensioned bar (3) is pulled till the established preload value. At this moment this load is withstand by the fragile bolt (2), the pre-tensioned bar and by the contrast ring (4). Because of the elastic movement of the entire system the pre-load on the specimen have to be checked and ruled by the actuator (8).

(iii) A mechanical system permits to continue to load the fragile bolt (2) without any influence on the rest of the measurement apparatus. In the 3D-MHB, the rupture of the fragile bolt gives rise to a rectangular square pulse propagating to the input bar (5) and output bar (7) and dynamically loading the specimen (6) until fracture.

(iv) The strain gages on the input and outputconfinement bar record the incident, reflected and transmitted waves that allow the reconstruction of the equivalent stress-strain curves in the analytical way.

In Fig. 3 the experimental set-up is shown.

\section{Results}

The critical point of the dynamic compression test with the $2 \mathrm{D}-\mathrm{MHB}$ is that common to all compression tests, that means it is affected by the variability of the boundary conditions at the interface loading member(bar)/specimen causing a remarkable dispersion of the test results. This high variability is due to the combined effects of contact between the specimen and the bars. Non-perfect shape of the specimen (such as imperfection of flatness and parallelism of the specimen faces) induces to a premature failure with anomalous crack paths. Also the contact

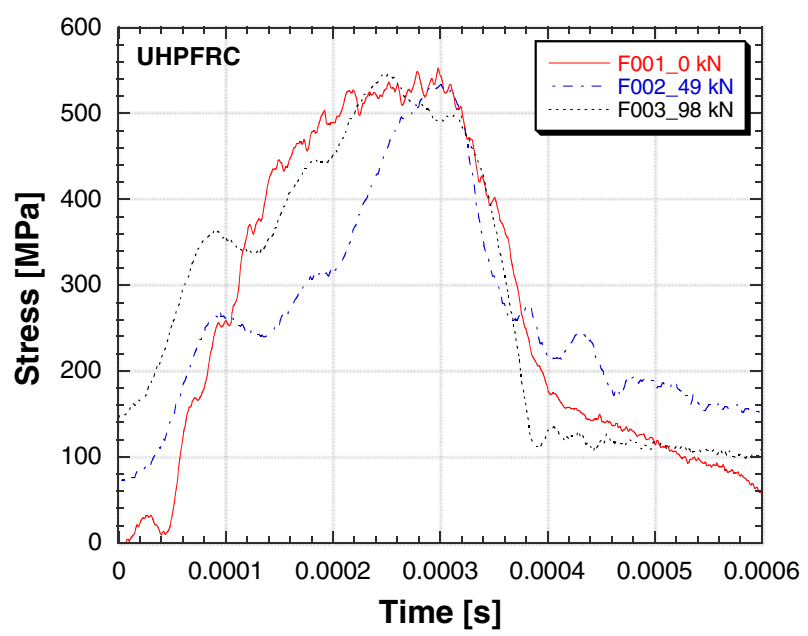

Figure 5. Stress versus time curves of UHPFRC with different pre-loading states.
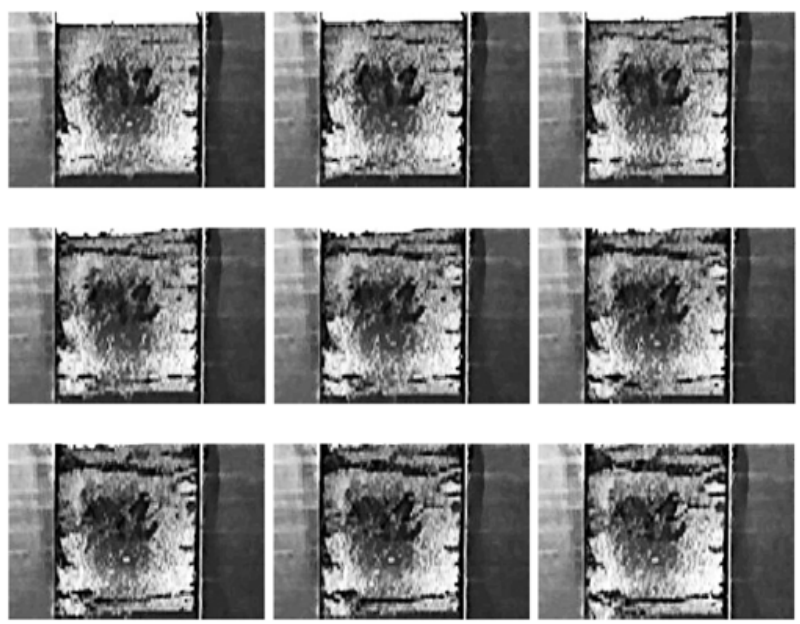

Figure 6. Photos of the UHPC specimen during the dynamic test with $0 \mathrm{kN}$ pre-loading.

friction between bar surface and specimen surface has an influence on the transversal deformation of the specimen contributing to the above-mentioned variability of the test results. Eventual presence of interface layers (such as copper or grease) between specimen and the bars can reduce these problems, but this fact introduces an additional variable that must be taken into account in the analysis.

As first application with the new 3D-MHB apparatus has been chosen the study of the influence of the quasistatic preload on the dynamic compressive behaviour of the UHPC and the preliminary results are presented. The preliminary testing campaign consisted of a series of dynamic compressive tests performed varying the static pre-stress condition of the specimen. At least three tests for each pre-stress condition were carried out. Before the dynamic test quasi-static tests were performed. The average failure load in the quasi-static tests was $118.7 \pm$ $6.2 \mathrm{kN}$. Two preload levels of $1 / 3$ and $2 / 3$ of the failure load were selected. The dynamic tests were carried out at the same velocity imposing $500 \mathrm{kN}$ in the pre-tensioned bar giving a stress rate of $1600 \mathrm{GPa} / \mathrm{s}$. The input and out bars 

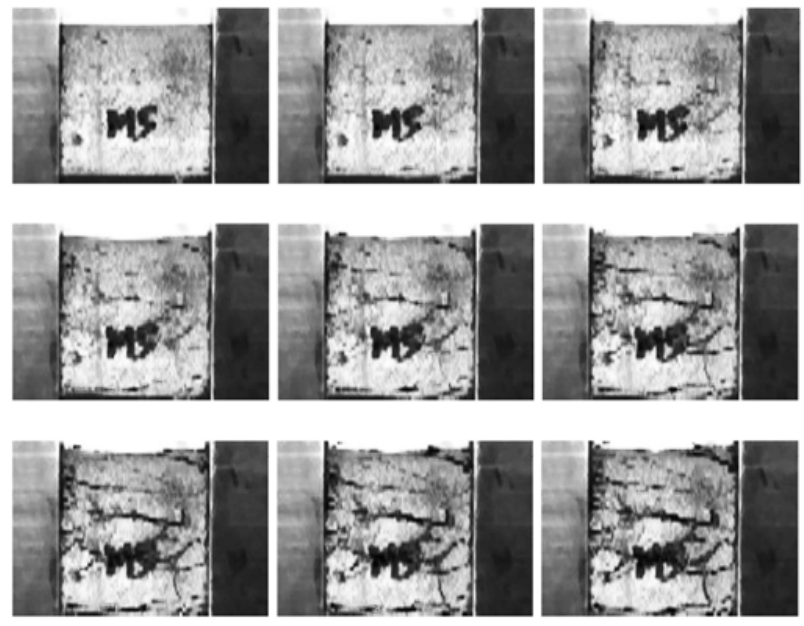

Figure 7. Photos of the UHPC specimen during the dynamic test with $40 \mathrm{kN}$ pre-loading.
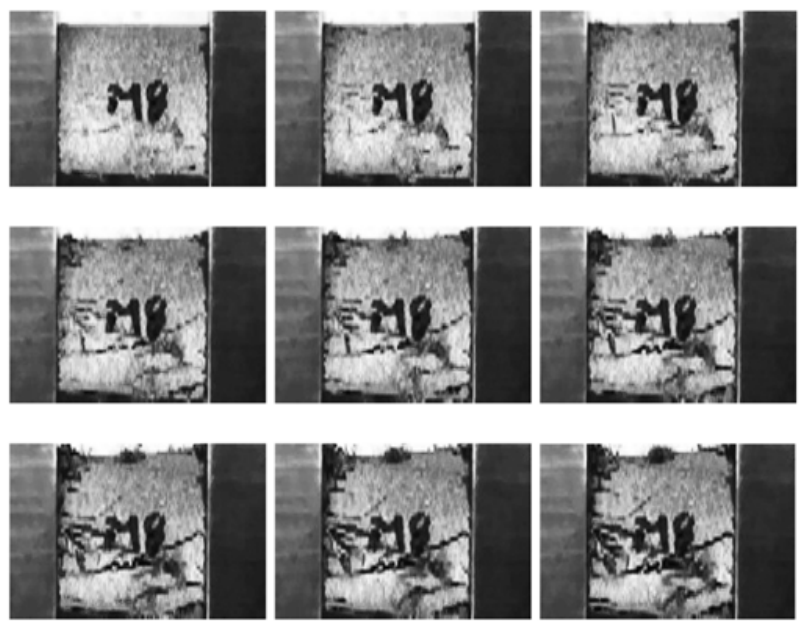

Figure 8. Photos of the UHPC specimen during the dynamic test with $80 \mathrm{kN}$ pre-loading.

were instrumented by strain gauges in order to obtain strain history. These measurements were acquired by means of HBM-GEN2 data acquisition system with high sampling rates (1 Msample/s). The results in terms of maximum strength are collected in Table 2.

Figure 4 shows stress versus time curves of the performed tests. The standard deviation decreases with increasing the pre-load conditions. This means that the preload decreases the importance of the boundary conditions role.

Stress gradient is the same for all tests till the firsts $60 \mu$ s. After that time the slope of the stress versus time curve changes. This is due to the presence of the activated cracks during the pre-load [8]. In fact, stress rate as well as the number of activated cracks is a critical factor to enucleate the cracking process that induces specimen failure. The tests with higher static pre-loading value show a more uniform response of the material, but the strength was lower because of damage by the static pre-loading. This investigation was enlarged to few specimens of Ultra High Performance Fibre Reinforced Concrete (UHPFRC) to better understand this behaviour. Indeed the UHPFRC was pre-loaded with the same ratios of $1 / 3$ and $2 / 3$ of quasi-static failure load corresponding to $49 \mathrm{kN}$ and $98 \mathrm{kN}$. In this case the UHPFRC presents not marked differences as the UHPC materials as shown in Fig. 5.

Figures 6, 7 and 8 shows some photos of the UHPC specimens during the failure process for $0 \mathrm{kN}, 40 \mathrm{kN}$ and $80 \mathrm{kN}$ pre-loading, respectively. The photos were acquired by IDT Y4-S3 fast digital camera at 53'000 frame/s highlighting the different crack paths.

\section{Conclusion}

The influence of the preloading state on the compressive response of UHPC has been analysed by means of a new apparatus called 3D-MHB.

These preliminary results underline the critical influence of the boundary condition on the material response in compression; such critical influence shall be reduced by increasing planarity and parallelism of the specimen surfaces and the precision of the specimen/bar set-up.

The contact surfaces of the specimen shall be conditioned with special greases reducing friction effects. Increasing the pre-load state of the material a damage state can be induced especially if the pre-load is over the half of the failure quasi-static values. For pre-load lower, an effects homogenisation in the interface between specimen and experimental apparatus is obtained.

The new apparatus has been funded in the frame of the R'Equip program of the Swiss National Sciences Foundation with the Project SNSF No. 206021_128734 (2011).

These first tests have been supported by armasuisse- Science and Technology of the Swiss Federal Department of Defence, Civil Protection and Sport in the frame of the project "Protection of Infrastructure Elements from the effects of IEDs and Blast Charges".

\section{References}

[1] A. Caverzan, E. Cadoni, M. di Prisco, Mech. Mat. 59, 87-109 (2013).

[2] A. Caverzan, E. Cadoni, M. di Prisco, Int. J. Imp. Engng 45, 28-38(2012).

[3] D. Asprone, E. Cadoni, A. Prota, ACI Struct. J. 106, 523-529 (2009).

[4] D. Asprone, E. Cadoni, A. Prota, ACI Struct. J. 106, 106-113 (2009).

[5] E. Cadoni, C. Albertini, Modified Hopkinson Bar Technologies Applied to the High Strain Rate of Rocks, in Advances in Rock Dynamics and Applications, Y. Zhou, J. Zhao eds. (Taylor \& Francis, 2011), 4, 79-104.

[6] J. Zhao, E. Cadoni, Triaxially Compressed Hopkinson Bar (TriHB) for Geomaterial and Construction Material Testing, Project SNSF No. 206021_128734 (2011).

[7] E. Cadoni, D. Forni, EPJ-ST (to be published) (2015).

[8] G. Riganti, E. Cadoni, Mat. Des. 57, 156-167 (2014). 